\title{
A Functional Equation Originating from Elliptic Curves
}

\author{
Won-Gil Park ${ }^{1}$ and Jae-Hyeong Bae ${ }^{2}$ \\ ${ }^{1}$ National Institute for Mathematical Sciences, 385-16 Doryong-Dong, Yuseong-Gu, \\ 305-340 Daejeon, South Korea \\ ${ }^{2}$ College of Liberal Arts, Kyung Hee University, 449-701 Yongin, South Korea
}

Correspondence should be addressed to Jae-Hyeong Bae, jhbae@khu.ac.kr

Received 17 November 2007; Revised 1 February 2008; Accepted 5 April 2008

Recommended by John Rassias

We obtain the general solution and the stability of the functional equation $f(x+y+z, u+v+$ $w)+f(x+y-z, u+v+w)+2 f(x, u-w)+2 f(y, v-w)=f(x+y, u+w)+f(x+y, v+$ $w)+f(x+z, u+w)+f(x-z, u+v-w)+f(y+z, v+w)+f(y-z, u+v-w)$. The function $f(x, y)=x^{3}+a x+b-y^{2}$ having level curves as elliptic curves is a solution of the above functional equation.

Copyright (C) 2008 W.-G. Park and J.-H. Bae. This is an open access article distributed under the Creative Commons Attribution License, which permits unrestricted use, distribution, and reproduction in any medium, provided the original work is properly cited.

\section{Introduction}

A graph of an equation of the form $y^{2}=x^{3}+a x+b$ is calledan elliptic curve [1], where $a$ and $b$ are constants. Since $f(x, y)=x^{3}+a x+b-y^{2}$ has level curves as elliptic curves, functional equations having the mapping $f(x, y)=x^{3}+a x+b-y^{2}$ as a solution are helpful to study cryptography and their applications.

Recently, Jun and Kim [2] solved the cubic functional equation

$$
\begin{aligned}
& g(x+y+z)+g(x+y-z)+2 g(x)+2 g(y) \\
& \quad=2 g(x+y)+g(x+z)+g(x-z)+g(y+z)+g(y-z) .
\end{aligned}
$$

We consider the quadratic functional equation

$$
g(x+y+z)+g(x-z)+g(y-z)=g(x+y-z)+g(x+z)+g(y+z),
$$


and the 2-dimensional vector variable functional equation

$$
\begin{aligned}
& f(x+y+z, u+v+w)+f(x+y-z, u+v+w)+2 f(x, u-w)+2 f(y, v-w) \\
&= f(x+y, u+w)+f(x+y, v+w)+f(x+z, u+w) \\
&+f(x-z, u+v-w)+f(y+z, v+w)+f(y-z, u+v-w)
\end{aligned}
$$

The function $g: \mathbb{R} \rightarrow \mathbb{R}$ given by $g(x):=a x^{3}+b x+c$ is a particular solution of (1.1), and the function $g: \mathbb{R} \rightarrow \mathbb{R}$ given by $g(x):=a x^{2}+b$ is a particular solution of (1.2). The function $f: \mathbb{R} \times \mathbb{R} \rightarrow \mathbb{R}$ given by $f(x, y):=x^{3}+a x+b-y^{2}$ is a particular solution of (1.3). The functional (1.3) is a mixed type of (1.1) and (1.2).

Problems on solutions or stability of various functional equations have been extensively investigated by a number of mathematicians [3-12].

In this paper, let $X, Y$, and $Z$ be real vector spaces. We find out the general solution and investigate the stability of (1.2) and (1.3).

\section{Solutions}

We find out the general solution of the functional (1.2) as follows.

Lemma 2.1. A mapping $g: X \rightarrow Y$ satisfies (1.2) if and only if there exists a symmetric biadditive mapping $S: X \times X \rightarrow Y$ such that

$$
g(x)=S(x, x)+g(0)
$$

for all $x \in X$.

Proof. We first assume that $g$ is a solution of (1.2). Let $h: X \rightarrow Y$ be a mapping given by $h(x):=g(x)-g(0)$ for all $x \in X$. Then, $h(0)=0$ and

$$
h(x+y+z)+h(x-z)+h(y-z)=h(x+y-z)+h(x+z)+h(y+z)
$$

for all $x, y, z \in X$. Putting $x=y=0$ in (2.2), $h$ is even. Replacing $z$ by $x+y$ in (2.2),

$$
h(2 x+2 y)+h(y)+h(x)=h(2 x+y)+h(x+2 y)
$$

for all $x, y \in X$. Taking $z=x$ in (2.2), we get that

$$
h(2 x+y)+h(x-y)=h(y)+h(2 x)+h(x+y)
$$

for all $x, y \in X$. Interchanging $x$ and $y$ in (2.4), we see that

$$
h(x+2 y)+h(x-y)=h(x)+h(2 y)+h(x+y)
$$

for all $x, y \in X$. By (2.3), (2.4), and (2.5), we obtain that

$$
\begin{aligned}
h(2 x+2 y) & =h(2 x+y)-h(y)+h(x+2 y)-h(x) \\
& =h(2 x)+h(x+y)-h(x-y)+h(2 y)+h(x+y)-h(x-y) \\
& =h(2 x)+h(2 y)+2 h(x+y)-2 h(x-y)
\end{aligned}
$$


for all $x, y \in X$. Letting $y=x$ in (2.6), we get that

$$
h(2 x)=4 h(x)
$$

for all $x \in X$. By (2.6) and (2.7), we obtain that

$$
h(x+y)+h(x-y)=2 h(x)+2 h(y)
$$

for all $x, y \in X$. By [13], there exists a symmetric biadditive mapping $S: X \times X \rightarrow Y$ such that $h(x)=S(x, x)$ for all $x \in X$.

Conversely, we assume that there exists a symmetric biadditive mapping $S: X \times X \rightarrow Y$ such that $g(x)=S(x, x)+g(0)$ for all $x \in X$. Since $S$ is biadditive,

$$
\begin{aligned}
g(x+ & y+z)+g(x-z)+g(y-z) \\
& =S(x+y+z, x+y+z)+S(x-z, x-z)+S(y-z, y-z)+3 g(0) \\
& =2[S(x, x)+S(x, y)+S(y, y)]+3 S(z, z)+3 g(0) \\
& =S(x+y-z, x+y-z)+S(x+z, x+z)+S(y+z, y+z)+3 g(0) \\
& =g(x+y-z)+g(x+z)+g(y+z)
\end{aligned}
$$

for all $x, y, z \in X$.

Example 2.2. Let $r / s$ be a rational number, where $r$ and $s$ are integers with $\operatorname{gcd}(r, s)=1$. Define $H(r / s):=\max \{|r|,|s|\}$ and $g(r / s):=\log H(r / s)$. Let $\mathbb{Q}$ be the set of rational numbers and let $E(\mathbb{Q}):=\{\infty\} \cup\left\{(x, y) \in \mathbb{Q} \times \mathbb{Q} \mid y^{2}=x^{3}+a x+b\right\}$ be an elliptic curve over $\mathbb{Q}$. The addition in $E(\mathbb{Q})$ is given in $[1]$. The height function $h: E(\mathbb{Q}) \rightarrow[0, \infty)$ is defined by

$$
h(x, y)=g(x), \quad h(\infty)=0
$$

for all $(x, y) \in E(\mathbb{Q})$. The canonical height function $\widehat{h}: E(\mathbb{Q}) \rightarrow[0, \infty)$ given by

$$
\widehat{h}(P)=\frac{1}{2} \lim _{n \rightarrow \infty} \frac{1}{4^{n}} h\left(2^{n} P\right)
$$

is a solution of (1.2).

We find out the general solution of the functional (1.3) as follows.

Theorem 2.3. A mapping $f: X \times Y \rightarrow Z$ satisfies (1.3) if and only if there exist a symmetric multiadditive mapping $S_{1}: X \times X \times X \rightarrow Z$, an additive mapping $A: X \rightarrow Z$, and a symmetric biadditive mapping $S_{2}: Y \times Y \rightarrow Z$ such that

$$
f(x, y)=S_{1}(x, x, x)+A(x)+f(0,0)+S_{2}(y, y)
$$

for all $x \in X$ and all $y \in Y$. 
Proof. We first assume that $f$ satisfies (1.3). Define $f_{1}: X \rightarrow Z$ and $f_{2}: Y \rightarrow Z$ by $f_{1}(x):=$ $f(x, 0)$ for all $x \in X$ and $f_{2}(y):=f(0, y)$ for all $y \in Y$. Then, $f_{1}$ satisfies (1.1) and $f_{2}$ satisfies (1.2). By [2], there exist a symmetric multiadditive mapping $S_{1}: X \times X \times X \rightarrow Z$ and an additive mapping $A: X \rightarrow Z$ such that

$$
f_{1}(x)=S_{1}(x, x, x)+A(x)+f_{1}(0)
$$

for all $x \in X$. By Lemma 2.1, there exists a symmetric biadditive mapping $S_{2}: Y \times Y \rightarrow Z$ such that

$$
f_{2}(y)=S_{2}(y, y)+f_{2}(0)
$$

for all $y \in Y$. Note that $f_{1}(0)=f_{2}(0)=f(0,0)$ and that $f(0,0) \neq 0$ in general. Let $k: X \times Y \rightarrow Z$ be a mapping given by $k(x, y):=f(x, y)-f(0,0)$ for all $x \in X$ and all $y \in Y$. Then, $k(0,0)=0$ and

$$
\begin{aligned}
& k(x+y+z, u+v+w)+k(x+y-z, u+v+w)+2 k(x, u-w)+2 k(y, v-w) \\
&= k(x+y, u+w)+k(x+y, v+w)+k(x+z, u+w) \\
&+k(x-z, u+v-w)+k(y+z, v+w)+k(y-z, u+v-w)
\end{aligned}
$$

for all $x, y, z \in X$ and all $u, v, w \in Y$.

Since $k(0, u)=S_{2}(u, u)$ for all $u \in Y, k(0,-u)=k(0, u)$ and $k(0,2 u)=4 k(0, u)$ for all $u \in Y$. Letting $y=z=u=v=0$ in (2.15), we get

$$
k(x, w)=k(x,-w)
$$

for all $x \in X$ and all $w \in Y$. Putting $y=z=0$ in (2.15), we get

$$
\begin{aligned}
& 2 k(x, u+v+w)+2 k(x, u-w)+2 k(0, v-w) \\
& \quad=2 k(x, u+w)+k(x, v+w)+k(0, v+w)+k(x, u+v-w)+k(0, u+v-w)
\end{aligned}
$$

for all $x \in X$ and all $u, v, w \in Y$. Setting $v=w=-u$ in (2.17) and using (2.16),

$$
k(x, u)+k(x, 2 u)=2 k(x, 0)+5 k(0, u)
$$

for all $x \in X$ and all $u \in Y$. Taking $v=-w=-u$ in (2.17) and using (2.16),

$$
k(x, u)+k(x, 0)+7 k(0, u)=2 k(x, 2 u)
$$

for all $x \in X$ and all $u \in Y$. By (2.18) and (2.19), $k(x, u)=k(x, 0)+k(0, u)$ for all $x \in X$ and all $u \in Y$. Hence, we obtain that

$$
\begin{aligned}
f(x, y) & =k(x, y)+f(0,0) \\
& =k(x, 0)+k(0, y)+f(0,0) \\
& =f_{1}(x)-f_{1}(0)+f_{2}(y)-f_{2}(0)+f(0,0) \\
& =S_{1}(x, x, x)+A(x)+f(0,0)+S_{2}(y, y)
\end{aligned}
$$

for all $x \in X$ and all $y \in Y$. 
Conversely, we assume that there exist a symmetric multiadditive mapping $S_{1}: X \times X \times$ $X \rightarrow Z$, an additive mapping $A: X \rightarrow Z$, and a symmetric biadditive mapping $S_{2}: Y \times Y \rightarrow Z$ such that

$$
f(x, y)=S_{1}(x, x, x)+A(x)+f(0,0)+S_{2}(y, y)
$$

for all $x \in X$ and all $y \in Y$. Since $S_{1}$ is symmetric multiadditive and $S_{2}$ is symmetric biadditive,

$$
\begin{aligned}
f(x+y & +z, u+v+w)+f(x+y-z, u+v+w)+2 f(x, u-w)+2 f(y, v-w) \\
= & S_{1}(x+y+z, x+y+z, x+y+z)+S_{1}(x+y-z, x+y-z, x+y-z) \\
& +2 S_{1}(x, x, x)+2 S_{1}(y, y, y)+A(x+y+z)+A(x+y-z) \\
& +2 A(x)+2 A(y)+2 S_{2}(u+v+w, u+v+w) \\
& +2 S_{2}(u-w, u-w)+2 S_{2}(v-w, v-w)+6 f(0,0) \\
= & 4 S_{1}(x, x, x)+4 S_{1}(y, y, y)+6 S_{1}(x, x, y)+6 S_{1}(x, y, y)+6 S_{1}(x, z, z) \\
& +6 S_{1}(y, z, z)+4 A(x)+4 A(y)+4 S_{2}(u, u)+4 S_{2}(u, v)+4 S_{2}(v, v) \\
& +6 S_{2}(w, w)+6 f(0,0) \\
= & 2 S_{1}(x+y, x+y, x+y)+S_{1}(x+z, x+z, x+z)+S_{1}(x-z, x-z, x-z) \\
& +S_{1}(y+z, y+z, y+z)+S_{1}(y-z, y-z, y-z) \\
& +2 A(x+y)+A(x+z)+A(x-z)+A(y+z)+A(y-z) \\
& +2 S_{2}(u+w, u+w)+2 S_{2}(v+w, v+w)+2 S_{2}(u+v-w, u+v-w) \\
& +6 f(0,0) \\
= & f(x+y, u+w)+f(x+y, v+w)+f(x+z, u+w)+f(x-z, u+v-w) \\
& +f(y+z, v+w)+f(y-z, u+v-w)
\end{aligned}
$$

for all $x, y, z \in X$ and all $u, v, w \in Y$.

Example 2.4. Let $X$ and $Y$ be the vector spaces $M_{3}(\mathbb{C})$ and $M_{2}(\mathbb{C})$, respectively. Consider a function $f: X \times Y \rightarrow \mathbb{C}$ given by $f(x, y):=\operatorname{det}(x)+\operatorname{det}(y)$ for all $x \in X$ and all $y \in Y$. One can easily verify that $f$ satisfies (1.3). By Theorem 2.3, there exist a symmetric multiadditive mapping $S_{1}: X \times X \times X \rightarrow \mathbb{C}$, an additive mapping $A: X \rightarrow \mathbb{C}$, and a symmetric biadditive mapping $S_{2}: Y \times Y \rightarrow \mathbb{C}$ such that

$$
f(x, y)=S_{1}(x, x, x)+A(x)+f(0,0)+S_{2}(y, y)
$$

for all $x \in X$ and all $y \in Y$. In fact,

$$
\begin{aligned}
& S_{1}(x, y, z)=\frac{1}{24}[\operatorname{det}(x+y+z)+\operatorname{det}(x-y-z)-\operatorname{det}(x+y-z)-\operatorname{det}(x-y+z)], \\
& A(x)=f(0,0)=0, \\
& S_{2}(u, v)=\frac{1}{4}[\operatorname{det}(u+v)-\operatorname{det}(u-v)]
\end{aligned}
$$

for all $x, y, z \in X$ and all $u, v \in Y$ 


\section{Stability}

From now on, let $Y$ be a Banach space, and let $\varphi: X^{3} \rightarrow[0, \infty)$ be a function satisfying

$$
\tilde{\varphi}(x, y, z):=\sum_{j=0}^{\infty} \frac{1}{4^{j+1}} \varphi\left(2^{j} x, 2^{j} y, 2^{j} z\right)<\infty
$$

for all $x, y, z \in X$.

Lemma 3.1. Let $g: X \rightarrow Y$ be a mapping such that

$$
\|g(x+y+z)+g(x-z)+g(y-z)-g(x+y-z)-g(x+z)-g(y+z)\| \leq \varphi(x, y, z)
$$

for all $x, y, z \in X$. Then, there exists a unique quadratic mapping $G: X \rightarrow Y$ satisfying (1.2) such that

$$
\|g(x)-G(x)\| \leq \tilde{\varphi}\left(\frac{x}{2}, \frac{x}{2}, x\right)+2 \widetilde{\varphi}\left(\frac{x}{2}, \frac{x}{2}, \frac{x}{2}\right)+2 \widetilde{\varphi}\left(0,0, \frac{x}{2}\right)+\frac{1}{3}[\varphi(0,0,0)+\|g(0)\|]
$$

for all $x \in X$. The mapping $G$ is given by $G(x):=\lim _{j \rightarrow \infty} \frac{1}{4^{j}} g\left(2^{j} x\right)$ for all $x \in X$.

Proof. Letting $x=y=0$ in (3.2), we have

$$
\|g(-z)-g(z)\| \leq \varphi(0,0, z)
$$

for all $z \in X$.

Replacing $z$ by $x+y$ in (3.2), we get

$$
\|g(2 x+2 y)+g(-y)+g(-x)-g(0)-g(2 x+y)-g(x+2 y)\| \leq \varphi(x, y, x+y)
$$

for all $x, y \in X$. Taking $z=x$ in (3.2), we see that

$$
\|g(2 x+y)+g(0)+g(y-x)-g(y)-g(2 x)-g(x+y)\| \leq \varphi(x, y, x)
$$

for all $x, y \in X$. Interchanging $x$ and $y$ in (3.6), we see that

$$
\|g(x+2 y)+g(0)+g(x-y)-g(x)-g(2 y)-g(x+y)\| \leq \varphi(y, x, x)
$$

for all $x, y \in X$. By (3.5), (3.6), and (3.7),

$$
\begin{aligned}
\| g(2 x & +2 y)-2 g(x+y)-g(2 x)-g(2 y) \| \\
\leq & \varphi(x, y, x+y)+\varphi(x, y, x)+\varphi(y, x, x) \\
\quad & +\|g(-x)+g(x)\|+\|g(-y)-g(y)\|+\|g(y-x)-g(x-y)\|+\|g(0)\| \\
\leq & \varphi(x, y, x+y)+\varphi(x, y, x)+\varphi(y, x, x)+\varphi(0,0, x)+\varphi(0,0, y)+\varphi(0,0, x-y)+\|g(0)\|
\end{aligned}
$$

for all $x, y \in X$. Putting $y=x$ in the above inequality, we get

$$
\|g(4 x)-4 g(2 x)\| \leq \varphi(x, x, 2 x)+2 \varphi(x, x, x)+2 \varphi(0,0, x)+\varphi(0,0,0)+\|g(0)\|
$$


for all $x \in X$. So,

$$
\left\|g(x)-\frac{1}{4} g(2 x)\right\| \leq \frac{1}{4} \varphi^{*}(x)
$$

where

$$
\varphi^{*}(x):=\varphi\left(\frac{x}{2}, \frac{x}{2}, x\right)+2 \varphi\left(\frac{x}{2}, \frac{x}{2}, \frac{x}{2}\right)+2 \varphi\left(0,0, \frac{x}{2}\right)+\varphi(0,0,0)+\|g(0)\|
$$

for all $x \in X$.

Thus, we obtain

$$
\left\|\frac{1}{4^{j}} g\left(2^{j} x\right)-\frac{1}{4^{j+1}} g\left(2^{j+1} x\right)\right\| \leq \frac{1}{4^{j+1}} \varphi^{*}\left(2^{j} x\right)
$$

for all $x \in X$ and all $j$. For given integers $l, m(0 \leq l<m)$, we get

$$
\left\|\frac{1}{4^{l}} g\left(2^{l} x\right)-\frac{1}{4^{m}} g\left(2^{m} x\right)\right\| \leq \sum_{j=l}^{m-1} \frac{1}{4^{j+1}} \varphi^{*}\left(2^{j} x\right)
$$

for all $x \in X$. By (3.13), the sequence $\left\{\left(1 / 4^{j}\right) g\left(2^{j} x\right)\right\}$ is a Cauchy sequence for all $x \in X$. Since $Y$ is complete, the sequence $\left\{\left(1 / 4^{j}\right) g\left(2^{j} x\right)\right\}$ converges for all $x \in X$. Define $G: X \rightarrow Y$ by

$$
G(x):=\lim _{j \rightarrow \infty} \frac{1}{4^{j}} g\left(2^{j} x\right)
$$

for all $x \in X$. By (3.2), we have

$$
\begin{gathered}
\| \frac{1}{4^{j}}\left[g\left(2^{j}(x+y+z)\right)+g\left(2^{j}(x-z)\right)+g\left(2^{j}(y-z)\right)-g\left(2^{j}(x+y-z)\right)\right. \\
\left.-g\left(2^{j}(x+z)\right)-g\left(2^{j}(y+z)\right)\right] \| \leq \frac{1}{4^{j}} \varphi\left(2^{j} x, 2^{j} y, 2^{j} z\right)
\end{gathered}
$$

for all $x, y, z \in X$ and all $j$. Letting $j \rightarrow \infty$ and using (3.1), we see that $G$ satisfies (1.2). Setting $l=0$ and taking $m \rightarrow \infty$ in (3.13), one can obtain the inequality (3.3). If $H: X \rightarrow Y$ is another quadratic mapping satisfying (1.2) and (3.3), we obtain

$$
\begin{aligned}
&\|G(x)-H(x)\|= \frac{1}{4^{n}}\left\|G\left(2^{n} x\right)-H\left(2^{n} x\right)\right\| \\
& \leq \frac{1}{4^{n}}\left\|G\left(2^{n} x\right)-g\left(2^{n} x\right)\right\|+\frac{1}{4^{n}}\left\|g\left(2^{n} x\right)-H\left(2^{n} x\right)\right\| \\
& \leq \frac{2}{4^{n}}\left[\widetilde{\varphi}\left(2^{n-1} x, 2^{n-1} x, 2^{n} x\right)+2 \widetilde{\varphi}\left(2^{n-1} x, 2^{n-1} x, 2^{n-1} x\right)+2 \widetilde{\varphi}\left(0,0,2^{n-1} x\right)\right. \\
&\left.\quad \quad+\frac{1}{3}[\varphi(0,0,0)+\|g(0)\|]\right] \longrightarrow 0 \quad \text { as } n \longrightarrow \infty
\end{aligned}
$$

for all $x \in X$. Hence, $G$ is a unique quadratic mapping, as desired. 
Example 3.2. If the height function $h$ in Example 2.2 satisfies

$$
\|h(P+Q+R)+h(P-R)+h(Q-R)-h(P+Q-R)-h(P+R)-h(Q+R)\| \leq c
$$

for all $P, Q, R \in E(\mathbb{Q})$, then there exists a unique quadratic function $\tilde{h}: E(\mathbb{Q}) \rightarrow[0, \infty)$ satisfying (1.2) such that

$$
\|h(P)-\tilde{h}(P)\| \leq 2 c
$$

for all $P \in E(\mathbb{Q})$.

Let $\psi: X^{3} \times Y^{3} \rightarrow[0, \infty)$ be a function satisfying

$$
\tilde{\psi}(x, y, z, u, v, w):=\sum_{j=0}^{\infty}\left[\frac{1}{2^{j+1}} \psi_{1}\left(2^{j} x, 2^{j} y, 2^{j} z\right)+\frac{1}{4^{j+1}} \psi_{2}\left(2^{j} u, 2^{j} v, 2^{j} w\right)\right]<\infty,
$$

where

$$
\psi_{1}(x, y, z):=\psi(x, y, z, 0,0,0), \quad \psi_{2}(u, v, w):=\psi(0,0,0, u, v, w)
$$

for all $x, y, z \in X$ and all $u, v, w \in Y$. Also, let $\widetilde{\psi}_{1}: X^{3} \rightarrow[0, \infty)$, and $\widetilde{\psi}_{2}: Y^{3} \rightarrow[0, \infty)$ be two functions given by

$$
\widetilde{\psi}_{1}(x, y, z):=\widetilde{\psi}(x, y, z, 0,0,0), \quad \widetilde{\psi}_{2}(u, v, w):=\widetilde{\psi}(0,0,0, u, v, w)
$$

for all $x, y, z \in X$ and all $u, v, w \in Y$.

Theorem 3.3. Let $f: X \times Y \rightarrow Z$ be a mapping such that

$$
\begin{aligned}
\| f(x+ & y+z, u+v+w)+f(x+y-z, u+v+w)+2 f(x, u-w)+2 f(y, v-w) \\
& -f(x+y, u+w)-f(x+y, v+w)-f(x+z, u+w)-f(x-z, u+v-w) \\
& -f(y+z, v+w)-f(y-z, u+v-w) \| \\
\leq & \psi(x, y, z, u, v, w)
\end{aligned}
$$

for all $x, y, z \in X$ and all $u, v, w \in Y$. Then, there exists a mapping $F: X \times Y \rightarrow Z$ satisfying (1.3) such that

$$
\begin{aligned}
\|f(x, y)-F(x, y)\| \leq & \psi(x, 0,0, y, 0,0)+\widetilde{\psi}_{1}\left(\frac{x}{2}, \frac{x}{2}, \frac{x}{2}\right)+\frac{1}{3} \widetilde{\psi}_{1}\left(x, \frac{x}{2}, \frac{x}{2}\right) \\
& +\widetilde{\psi}_{2}\left(\frac{y}{2}, \frac{y}{2}, y\right)+2 \widetilde{\psi}_{2}\left(\frac{y}{2}, \frac{y}{2}, \frac{y}{2}\right)+2 \widetilde{\psi}_{2}\left(0,0, \frac{y}{2}\right) \\
& +\frac{1}{3}\left[\psi_{2}(0,0,0)+\|f(0,0)\|\right]
\end{aligned}
$$

for all $x \in X$ and all $y \in Y$. The mapping $F$ is given by

$$
F(x, y):=\lim _{j \rightarrow \infty}\left[\frac{4^{j+1}-1}{3 \cdot 8^{j}} f\left(2^{j} x, 0\right)-\frac{4^{j}-1}{6 \cdot 8^{j}} f\left(2^{j+1} x, 0\right)+\frac{1}{4^{j}} f\left(0,2^{j} y\right)\right]-f(0,0)
$$

for all $x \in X$ and all $y \in Y$. 
Proof. Letting $y=z=v=w=0$ in (3.22), we have

$$
\|f(x, u)-f(x, 0)-f(0, u)+f(0,0)\| \leq \psi(x, 0,0, u, 0,0)
$$

for all $x \in X$ and all $u \in Y$.

Define $f_{1}, f_{2}: X \rightarrow Y$ by $f_{1}(x):=f(x, 0)$ and $f_{2}(u):=f(0, u)$ for all $x \in X$ and all $u \in Y$. Putting $u=v=w=0$ in (3.22), we have

$$
\begin{aligned}
& \| f_{1}(x+y+z)+f_{1}(x+y-z)+2 f_{1}(x)+2 f_{1}(y) \\
& \quad-2 f_{1}(x+y)-f_{1}(x+z)-f_{1}(x-z)-f_{1}(y+z)-f_{1}(y-z) \| \\
& \leq \psi_{1}(x, y, z)
\end{aligned}
$$

for all $x, y, z \in X$. Setting $x=y=z=0$ in (3.22), we have

$$
\left\|f_{2}(u+v+w)+f_{2}(u-w)+f_{2}(v-w)-f_{2}(u+v-w)-f_{2}(u+w)-f_{2}(v+w)\right\| \leq \psi_{2}(u, v, w)
$$

for all $u, v, w \in Y$.

By [2], there exists a cubic mapping $F_{1}: X \rightarrow Z$ satisfying (1.1) such that

$$
\left\|f_{1}(x)-F_{1}(x)\right\| \leq \widetilde{\psi}_{1}\left(\frac{x}{2}, \frac{x}{2}, \frac{x}{2}\right)+\frac{1}{3} \widetilde{\psi}_{1}\left(x, \frac{x}{2}, \frac{x}{2}\right)
$$

for all $x \in X$. By Lemma 3.1, there exists a quadratic mapping $F_{2}: Y \rightarrow Z$ satisfying (1.2) such that

$$
\left\|f_{2}(y)-F_{2}(y)\right\| \leq \widetilde{\psi}_{2}\left(\frac{y}{2}, \frac{y}{2}, y\right)+2 \widetilde{\psi}_{2}\left(\frac{y}{2}, \frac{y}{2}, \frac{y}{2}\right)+2 \widetilde{\psi}_{2}\left(0,0, \frac{y}{2}\right)+\frac{1}{3}\left[\psi_{2}(0,0,0)+\|f(0,0)\|\right]
$$

for all $y \in Y$.

If we define

$$
F(x, y):=F_{1}(x)+F_{2}(y)-f(0,0)
$$

for all $x \in X$ and all $y \in Y$, we conclude that

$$
\begin{aligned}
\|f(x, y)-F(x, y)\| \leq & \psi(x, 0,0, y, 0,0)+\widetilde{\psi}_{1}\left(\frac{x}{2}, \frac{x}{2}, \frac{x}{2}\right)+\frac{1}{3} \widetilde{\psi}_{1}\left(x, \frac{x}{2}, \frac{x}{2}\right) \\
& +\widetilde{\psi}_{2}\left(\frac{y}{2}, \frac{y}{2}, y\right)+2 \widetilde{\psi}_{2}\left(\frac{y}{2}, \frac{y}{2}, \frac{y}{2}\right)+2 \widetilde{\psi}_{2}\left(0,0, \frac{y}{2}\right) \\
& +\frac{1}{3}\left[\psi_{2}(0,0,0)+\|f(0,0)\|\right]
\end{aligned}
$$

for all $x \in X$ and all $y \in Y$.

Let $\varphi^{\prime}: X^{3} \rightarrow[0, \infty)$ be a function satisfying

$$
\sum_{j=0}^{\infty} 4^{j+1} \varphi^{\prime}\left(2^{-j} x, 2^{-j} y, 2^{-j} z\right)<\infty
$$

for all $x, y, z \in X$. For the function $\varphi^{\prime}$, we can obtain similar results to Lemma 3.1 and Theorem 3.3.

As a corollary, one can obtain a result when the control mapping is a summation of terms $\|\cdot\|^{p}$, in which $p$ is a suitable constant by referring to [14]. 


\section{References}

[1] L. C. Washington, Elliptic Curves: Number Theory and Cryptography, Discrete Mathematics and Its Applications, Chapman \& Hall/CRC, Boca Raton, Fla, USA, 2003.

[2] K.-W. Jun and H.-M. Kim, "Ulam stability problem for a mixed type of cubic and additive functional equation," Bulletin of the Belgian Mathematical Society. Simon Stevin, vol. 13, no. 2, pp. 271-285, 2006.

[3] T. Aoki, "On the stability of the linear transformation in Banach spaces," Journal of the Mathematical Society of Japan, vol. 2, pp. 64-66, 1950.

[4] J.-H. Bae and K.-W. Jun, "On the generalized Hyers-Ulam-Rassias stability of an n-dimensional quadratic functional equation," Journal of Mathematical Analysis and Applications, vol. 258, no. 1, pp. 183-193, 2001.

[5] Y.-S. Cho and H.-M. Kim, "Stability of functional inequalities with Cauchy-Jensen additive mappings," Abstract and Applied Analysis, vol. 2007, Article ID 89180, 13 pages, 2007.

[6] S. Czerwik, Functional Equations and Inequalities in Several Variables, World Scientific, River Edge, NJ, USA, 2002.

[7] S.-M. Jung, Hyers-Ulam-Rassias Stability of Functional Equations in Mathematical Analysis, Hadronic Press, Palm Harbor, Fla, USA, 2001.

[8] M. S. Moslehian, "Ternary derivations, stability and physical aspects," Acta Applicandae Mathematicae, vol. 100, no. 2, pp. 187-199, 2008.

[9] C. Park and J. Cui, "Generalized stability of $C^{*}$-ternary quadratic mappings," Abstract and Applied Analysis, vol. 2007, Article ID 23282, 6 pages, 2007.

[10] W.-G. Park and J.-H. Bae, “On a bi-quadratic functional equation and its stability," Nonlinear Analysis: Theory, Methods \& Applications, vol. 62, no. 4, pp. 643-654, 2005.

[11] W.-G. Park and J.-H. Bae, "A multidimensional functional equation having quadratic forms as solutions," Journal of Inequalities and Applications, vol. 2007, Article ID 24716, 8 pages, 2007.

[12] Th. M. Rassias, "On the stability of functional equations and a problem of Ulam," Acta Applicandae Mathematicae, vol. 62, no. 1, pp. 23-130, 2000.

[13] J. Aczél and J. Dhombres, Functional Equations in Several Variables, vol. 31 of Encyclopedia of Mathematics and Its Applications, Cambridge University Press, Cambridge, UK, 1989.

[14] Th. M. Rassias, "On the stability of the linear mapping in Banach spaces," Proceedings of the American Mathematical Society, vol. 72, no. 2, pp. 297-300, 1978. 


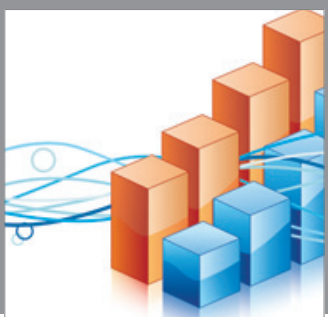

Advances in

Operations Research

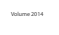

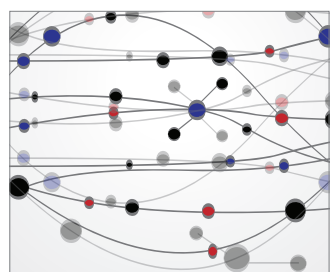

\section{The Scientific} World Journal
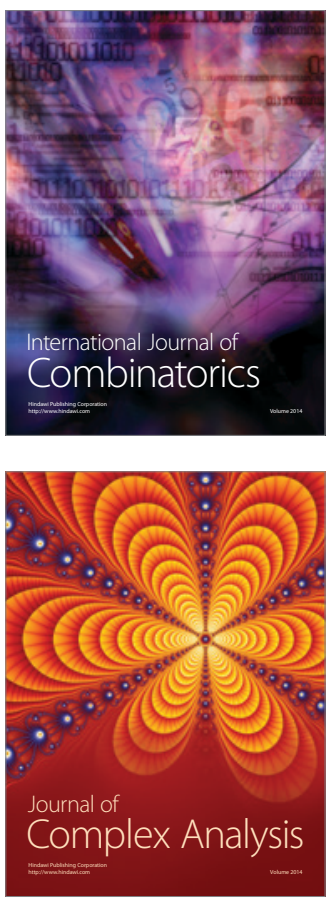

International Journal of

Mathematics and

Mathematical

Sciences
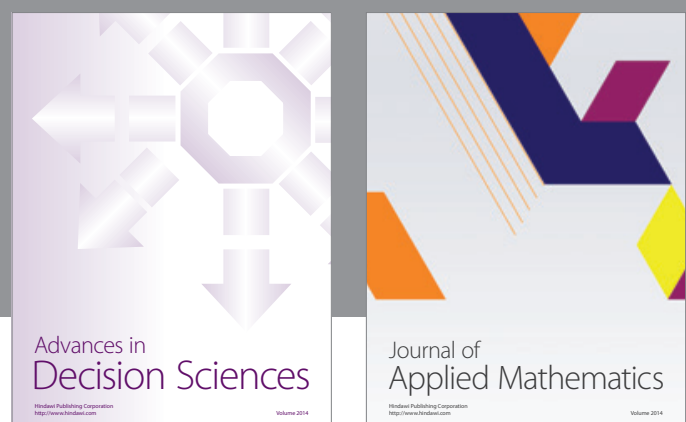

Journal of

Applied Mathematics
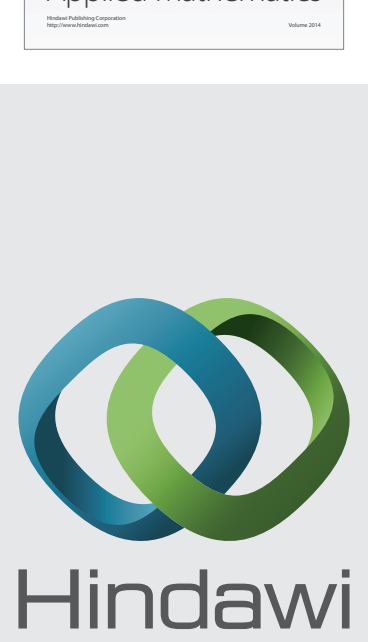

Submit your manuscripts at http://www.hindawi.com
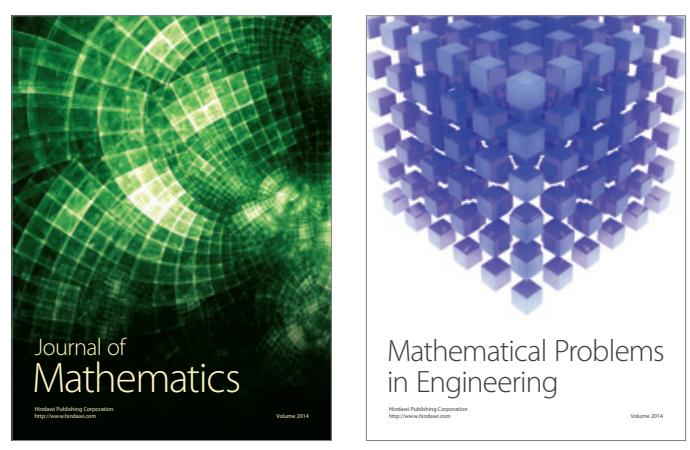

Mathematical Problems in Engineering
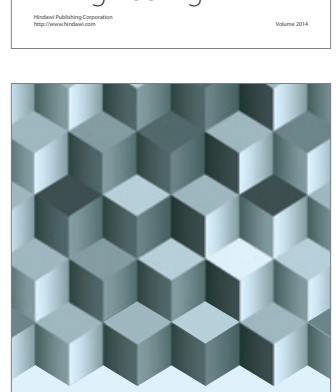

Journal of

Function Spaces
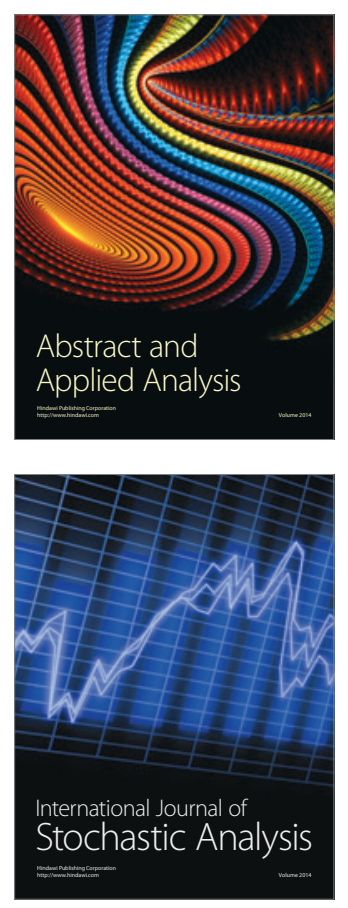

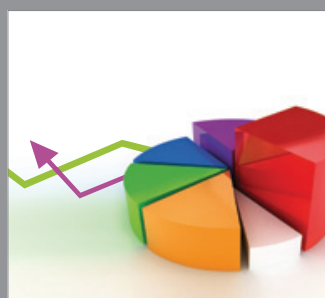

ournal of

Probability and Statistics

Promensencen
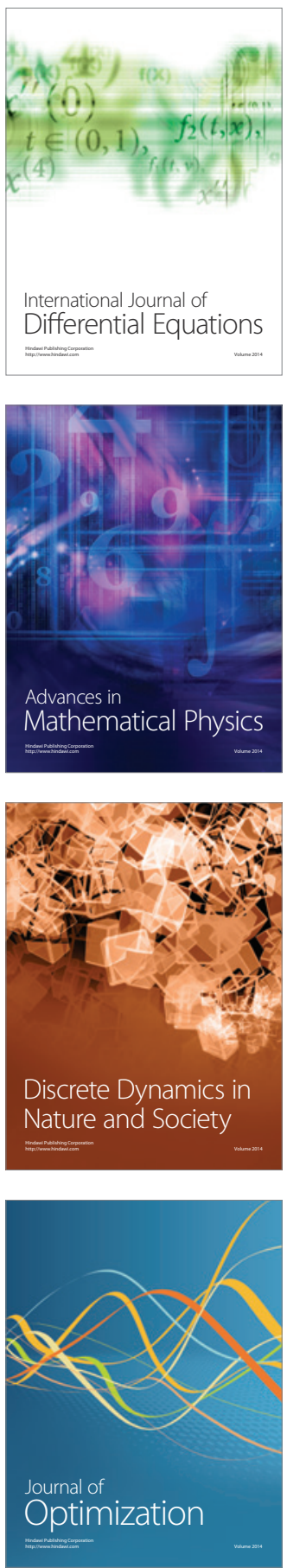